\title{
Effect of DEM Parameters on the Simulated Inter-particle Percolation of Pellets into Coke during Burden Descent in the Blast Furnace
}

\author{
Yaowei YU* and Henrik SAXÉN \\ Thermal and Flow Engineering Laboratory, Dept. of Chemical Engineering, Åbo Akademi University, Biskopsgatan 8, Fl-20500 \\ Åbo Finland. E-mail: yyu@abo.fi
}

(Received on September 21, 2011; accepted on December 19, 2011)

\begin{abstract}
Inter-particle percolation at the interface between the burden layers in the blast furnace influences the permeability in the lumpy zone, and, in particular, in the cohesive zone, where the iron-bearing materials start softening to finally melt. This paper presents a simulation study of the effect of particle properties on inter-particle percolation of small particles (pellets) into a layer of larger particles (coke) during burden descent in the blast furnace. An expanding experimental device in small scale was applied to mimic the conditions at burden descent in a shaft with growing radius, and results from these experiments were used as a reference for the simulations and to validate the computational results. The simulations, which were based on the discrete element method, studied the effect of factors such as friction and restitution coefficients, shear modulus, as well as pellet diameter on the extent of percolating particles. It was found that coke shape, pellet diameter, static friction and inter-particle rolling friction and restitution had a marked effect on the percolation, while rate of expansion of the device, density of pellet and shear modulus proved to be of minor importance.
\end{abstract}

KEY WORDS: discrete element method; size segregation and percolation; DEM parameters; blast furnace.

\section{Introduction}

Inter-particle percolation is a common phenomenon and it is of significance in many industrial situations, such as in powder metallurgy, pharmaceuticals, agriculture, glass and paint industries as well as in the ironmaking industry. Interparticle percolation has been investigated by means of various experimental and calculation methods in the past. Bridgwater and his colleagues ${ }^{1-3)}$ made pioneering research in studying experimentally inter-particle percolation, focusing on percolation velocity, residence time and radial distance distribution. Jha and Puri ${ }^{4)}$ studied percolation segregation of multi-size and multi-component particulate materials experimentally, including the effect of particle surface and strain rates. Richard et al. ${ }^{5)}$ proposed a Monte Carlo method to simulate the inter-particle percolation of a fine particle through a packing of monosize spheres. Lomine and Oger, $\left.{ }^{6}\right)$ in turn, performed experiments and discrete element method (DEM) simulation to analyze transport properties of particles through porous structure and analyzed the influence of the restitution coefficient and the size ratio between spheres. Rahman and $\mathrm{Zhu}^{7,8)}$ and Li et al. ${ }^{9)}$ used DEM to investigate percolation behavior, including percolation velocity, residence time distribution and radial dispersion under various conditions and found the simulation results consistent with the experiments reported by Bridgwater. Nevertheless, the cases studied were limited to launches of one or a small number of percolating particles.
In the ironmaking blast furnace the number of particles (mainly sinter/pellets and coke) charged is huge. Even though the charged volumes of iron bearing materials and coke are roughly equal, the considerably smaller size of pellet and sinter compared to coke means that there is a larger number of small particles that are susceptible to percolate into an underlying layer of the "packing particles" (coke). Furthermore, inter-particle percolation is present not only when the materials are charged, but also when the burden descends in the furnace. In the blast furnace shaft, the crosssectional area perpendicular to the central axis of the furnace increases from the throat to the belly, and the descending materials spread out to completely cover the cross-sectional area at any vertical position in the shaft. Along with this, individual particles move and rotate relative to each other, opening up new voids and closing others. Small particles can then percolate into these voids, causing further deformation of the burden layers. The inter-particle percolation naturally influences the permeability of the burden layers in the shaft, and, in particular, the cohesive zone where the iron-bearing phases start softening to finally melt. Even though many investigators ${ }^{10-16)}$ have studied burden descent in the blast furnace, inter-particle percolation has not attracted much interest.

Based on results reported earlier, where a device was developed for mimicking the conditions of a layer of pellets resting on a layer of coke under expansion and DEM simulations of the system were reported, this present work makes 
a deeper analysis of factors in the simulations that influence the percolation. Factors studied include the restitution coefficient between the particles, the sliding and rolling friction coefficients and the diameter ratio of the percolating and packing particles. The burden descent velocity and pellet charging method are also considered. Some conclusions concerning the inter-particle percolation segregation phenomena are proposed as well as on the usefulness of DEM simulations in investigations of complex flow of particles.

\section{Experimental System}

In order to investigate experimentally the percolation of pellets into an underlying coke layer, a device emulating the conditions during burden descent in the blast furnace was constructed, as shown in Fig. 1. The wall, inclined to represent the shaft, can move up and left (see large white arrow) with the lower horizontal bottom plate simultaneously moving left to mimic the increasing cross-sectional area during burden descent. Two layers of particles, coke on the bottom and pellets on the top (scaled down by 1:4 with respect to typical sizes used in the real process), are placed in the device, which is slowly expanded. On the bottom plate there are (black) rubber stripes (cf. Fig. 1), which guarantee that the lowest layer of particles will not slide on the bottom surface but move together with the expansion. The device was slowly expanded to finally reach a maximum expansion of $l_{\max }=200 \mathrm{~mm}$, and the particles were observed through the plexiglass throughout the expansion process. In addition, the height of the material bed was measured at four positions, with the relative widths of $1 / 8,3 / 8,5 / 8$ and $7 / 8$ (cf. triangles in Fig. 1). The pellets used in the experiments have a particle diameter of $3 \pm 1 \mathrm{~mm}$, while coke has the following four sizes (with corresponding mass fractions reported in parentheses): Coke1: $10-16 \mathrm{~mm}$ (47\%), Coke2: 16-18 mm (36\%) and Coke3: 20-25 mm (17\%). More details about the device and the experiments are given in Ref. 17).

\section{Discrete Element Method}

The translational and rotational motion of granular particles can be described by Newton's second law of motion

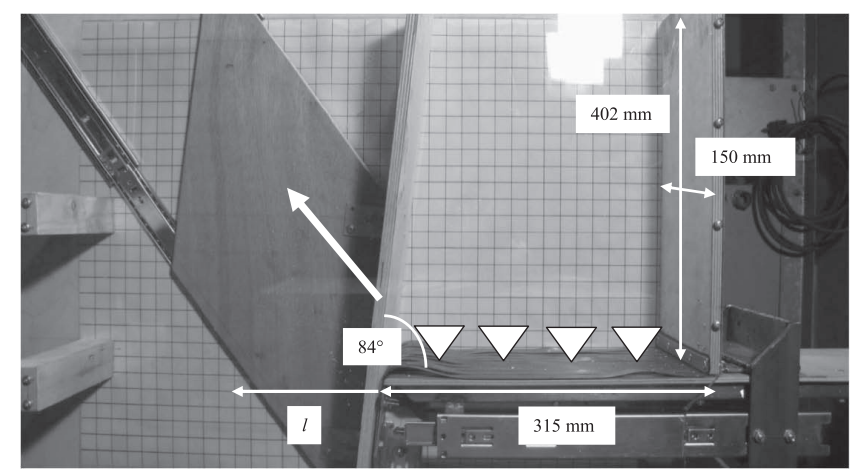

Fig. 1. Experimental device used to emulate the conditions under burden descent. ${ }^{17)}$ Arrowheads denote the dimensionless positions $1 / 8,3 / 8,5 / 8$ and $7 / 8$ (at the state of no expansion) where the bed height was measured, and $l$ the bed expansion. combined with a force-displacement correlation at the points of contact between the particles. The discrete element method (DEM) ${ }^{18)}$ applies an inter-particle contact model schematically depicted in Fig. 2: A spring and a dashpot correspond to the elastic and plastic nature of particles in the normal direction, while the model consists of a slider, a spring and a dashpot in the tangential direction. This gives the governing equations for a particle $(i)$ in interaction with another particles $(j)$ as ${ }^{19,20)}$

$$
\begin{array}{r}
m_{i} \frac{d \mathbf{u}_{i}}{d t}=\sum_{j=1}^{K}\left(\mathbf{F}_{\mathrm{cn}, i j}+\mathbf{F}_{\mathrm{dn}, i j}+\mathbf{F}_{\mathrm{ct}, i j}+\mathbf{F}_{\mathrm{dt}, i j}\right)+m_{i} \mathbf{g} \ldots \ldots .(1) \\
I_{i} \frac{\mathrm{d} \omega_{i}}{\mathrm{~d} t}=\sum_{j=1}^{K}\left(\mathbf{T}_{\mathrm{t}, i j}+\mathbf{T}_{\mathrm{r}, i j}\right) \ldots \ldots \ldots \ldots \ldots \ldots \ldots \ldots \ldots \ldots \ldots \ldots \ldots
\end{array}
$$

In Eqs. (1)-(2), $\mathbf{u}_{i}$, is the translational and $\omega_{i}$ the angular velocity, while $I_{i}$ is the moment of inertia of particle $i$. The forces acting on the particle are the inter-particle forces and the gravitational force, $m_{i} \mathbf{g}$. The formers include the normal contact force, $\mathbf{F}_{\mathrm{cn}, i j}$, and the tangential contact force, $\mathbf{F}_{\mathrm{ct}, i j}$, as well as the corresponding viscous damping forces, $\mathbf{F}_{\mathrm{dn}, i j}$ and $\mathbf{F}_{\mathrm{dt}, i j}$. The inter-particle forces, which depend on the normal and tangential deformations, $\delta_{\mathrm{n}}$ and $\delta_{\mathrm{t}}$, are summed over all the $K$ particles in contact with particle $i$. The torque acting on particle $i$ includes a component from tangential force, $\mathbf{T}_{\mathrm{t}, i j}$ and another from the rolling friction, $\mathbf{T}_{\mathrm{r}, i j}$. The hardness of the particles and the dashpot are related to Young's modulus and the coefficient of restitution, respectively, while the friction is expressed with a Coulomb-type friction law. The particle forces and torques (see the Appendix ${ }^{21-27)}$ ) give rise to particle motion, which is integrated from the differential Eqs. (1)-(2), simultaneously considering interaction with other particles and boundaries in the system. Due to the explicit integration method used and the large number of particles in close contact with each other, the time step has to be kept small and the computational effort of the method is considerable. The EDEM ${ }^{25}$ software was applied to simulate the motion of the particles in the experimental device, with the simplification that the depth of the simulated system was decreased from $150 \mathrm{~mm}$ to $80 \mathrm{~mm}$ to save compu-

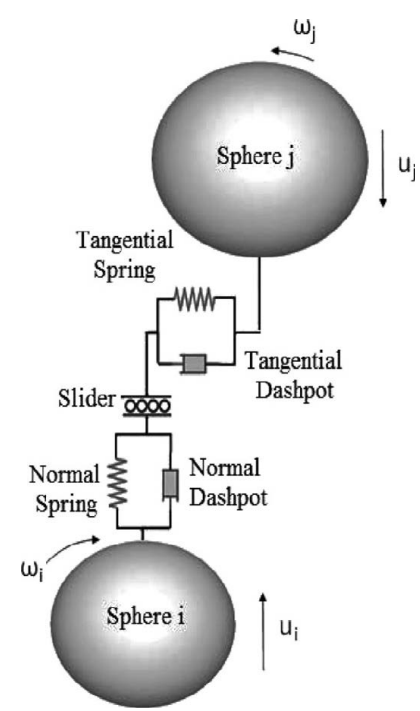

Fig. 2. Schematic illustration of the interaction between two particles, $i$ and $j$, in DEM. 
Table 1. DEM parameters used in base case in the simulations with pellets of one size and coke of three sizes (Coke1, Coke2 and Coke3). (Alternative values of number of pellets and pellet diameters are reported in parentheses.) Particle diameters correspond to average of diameters in the experiments. Low values of the shear modulus of coke and pellets were used to increase calculation speed. When changing one parameter, the others were kept at their base-case values.

\begin{tabular}{|c|c|}
\hline Parameter & Value \\
\hline \multicolumn{2}{|l|}{ Pellets } \\
\hline Number & $66294(104453,26197)$ \\
\hline Diameter, $D(\mathrm{~mm})$ & $3(2,4)$ \\
\hline Density, $\rho\left(\mathrm{kg} / \mathrm{m}^{3}\right)$ & 2284 \\
\hline Shear modulus, $G(\mathrm{MPa})$ & 10 \\
\hline Poisson's ratio, $E^{*}$ & 0.25 \\
\hline Coefficient of pellet-pellet static friction, $\mu_{\mathrm{s}}$ & 0.50 \\
\hline Coefficient of pellet-pellet rolling friction, $\mu_{\mathrm{r}}$ & 0.15 \\
\hline Coefficient of pellet-pellet restitution, $e$ & 0.60 \\
\hline Coefficient of pellet-coke static friction, $\mu_{\mathrm{s}}$ & 0.52 \\
\hline Coefficient of pellet-coke rolling friction, $\mu_{\mathrm{r}}$ & 0.10 \\
\hline Coefficient of pellet-coke restitution, $e$ & 0.75 \\
\hline \multicolumn{2}{|l|}{ Coke } \\
\hline Diameter of Coke1, $D(\mathrm{~mm})$ & 13 \\
\hline Number of Coke1 particles & 832 \\
\hline Diameter of Coke2, $D(\mathrm{~mm})$ & 18 \\
\hline Number of Coke 2 particles & 225 \\
\hline Diameter of Coke3, $D(\mathrm{~mm})$ & 22.5 \\
\hline Number of Coke3 particles & 54 \\
\hline Density, $\rho\left(\mathrm{kg} / \mathrm{m}^{3}\right)$ & 1050 \\
\hline Shear modulus, $G(\mathrm{MPa})$ & 2.2 \\
\hline Poisson's ratio, $E^{*}$ & 0.22 \\
\hline Coefficient of coke-coke static friction, $\mu_{\mathrm{s}}$ & 0.43 \\
\hline Coefficient of coke-coke rolling friction, $\mu_{\mathrm{r}}$ & 0.15 \\
\hline Coefficient of coke-coke restitution, $e$ & 0.85 \\
\hline \multicolumn{2}{|l|}{ Plexiglass } \\
\hline Density, $\rho\left(\mathrm{kg} / \mathrm{m}^{3}\right)$ & 1500 \\
\hline Shear modulus, $G(\mathrm{MPa})$ & 0.1 \\
\hline Poisson's ratio, $E^{*}$ & 0.4 \\
\hline Coefficient of wall-pellet static friction, $\mu_{\mathrm{s}}$ & 0.35 \\
\hline Coefficient of wall-pellet rolling friction, $\mu_{\mathrm{r}}$ & 0.10 \\
\hline Coefficient of wall-pellet restitution, $e$ & 0.20 \\
\hline Coefficient of wall-coke static friction, $\mu_{\mathrm{s}}$ & 0.41 \\
\hline Coefficient of wall-coke rolling friction, $\mu_{\mathrm{r}}$ & 0.09 \\
\hline Coefficient of wall-coke restitution, $e$ & 0.20 \\
\hline \multicolumn{2}{|l|}{ Bottom plate (rubber) } \\
\hline Density, $\rho\left(\mathrm{kg} / \mathrm{m}^{3}\right)$ & 1200 \\
\hline Shear modulus, $G(\mathrm{MPa})$ & 0.1 \\
\hline Poisson's ratio, $E^{*}$ & 0.45 \\
\hline Coefficient of rubber-coke static friction, $\mu_{\mathrm{s}}$ & 0.90 \\
\hline Coefficient of rubber-coke rolling friction, $\mu_{\mathrm{r}}$ & 0.34 \\
\hline Coefficient of rubber-coke restitution, $e$ & 0.10 \\
\hline Time step, $\Delta t(\mathrm{~s})$ & $0.14 \times 10^{-6}$ \\
\hline Rate of expansion of device, $w(\mathrm{~mm} / \mathrm{s})$ & 1.5 \\
\hline
\end{tabular}

tational time. The physical properties used for coke particles, pellet particles and plexiglass wall are reported in Table 1, where pellet and coke data were taken from the literature ${ }^{26,28)}$ with a constant time step.

\section{Results and Discussion}

\subsection{Basic Setup}

The experimental results ${ }^{17)}$ for a single coke or pellet layer revealed that with the expansion of the device, the left side of the burden surface declined and finally the surface of practically the whole bed showed an angle. For the coke, the declination of the surface profile was more pronounced while it was more regular for pellets. The overall behavior was described well by the DEM simulations with spherical particles ( $c f$. Table 1), which was found to have captured the essential features observed in the experiments. ${ }^{17)}$ Figure 3 illustrates experimental results (left) and corresponding DEM simulation (right) for a setup where a layer of pellets was placed on a layer of coke ${ }^{17)}$ where the panels correspond to different expansions of the device, starting (at the top) with no $(0 \mathrm{~mm})$ and ending (at the bottom) with maximum $(200 \mathrm{~mm})$ expansion. The extent of inter-particle percolation grows with the expansion of the device, in particular after the $150 \mathrm{~mm}$ expansion stage (fourth subfigure from top) has

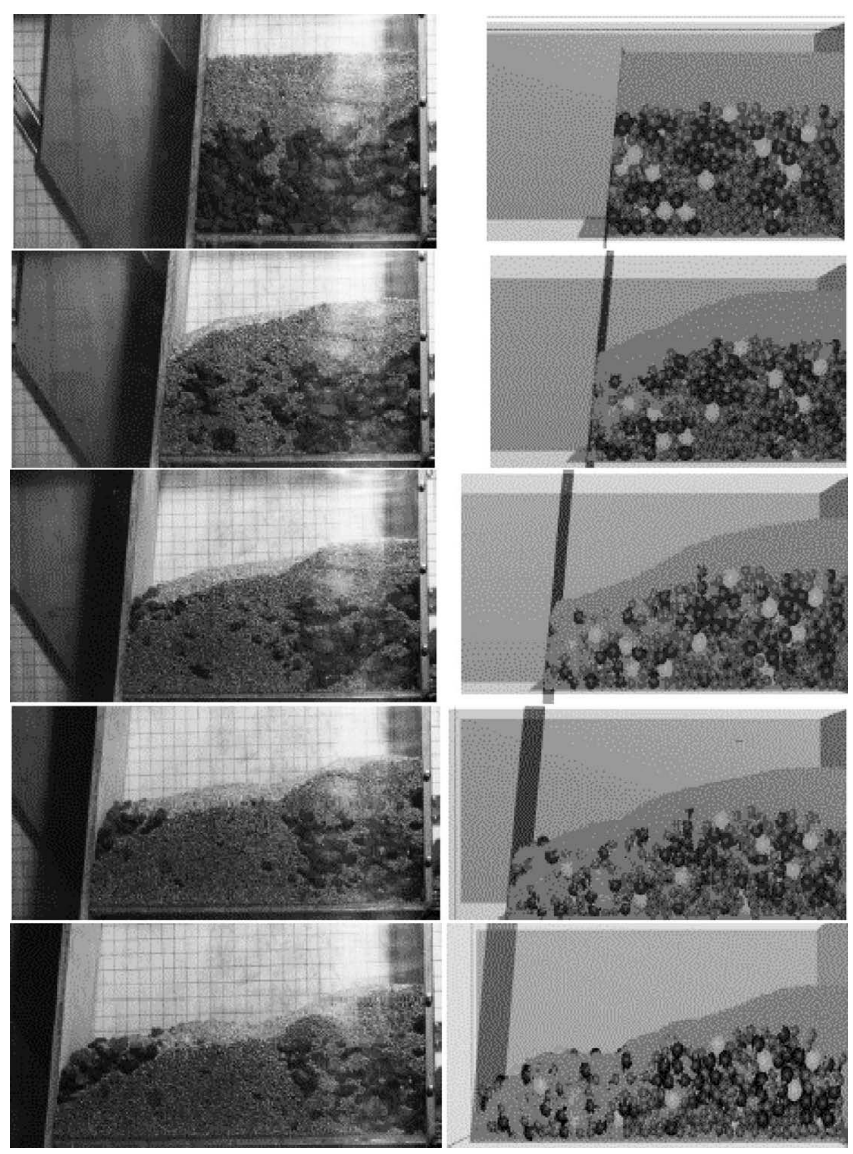

Fig. 3. Experimental (left) and DEM simulation (right) results for an expansion experiment with a pellet layer (initial height $62 \mathrm{~mm}$ ) on a coke layer (initial height $144 \mathrm{~mm}$ ). ${ }^{17)}$ Results are illustrated for a device expansion of $0 \mathrm{~mm}$ (top) to 200 $\mathrm{mm}$ (bottom) with an increment of $50 \mathrm{~mm}$. Coke fractions are illustrated in dark gray (small), black (intermediate) and white (large), while pellets are depicted in light gray. 
been reached. The pellet layer is seen to bend at the moving wall and the strong percolation of the pellets finally reveals the top parts of the coke bed. Interestingly, the pellet layer now shows a rather uniform slope in the device. Table 2 reports the experimentally measured and the simulated bed heights at four dimensionless bed widths $(1 / 8,3 / 8,5 / 8$, and $7 / 8$, cf. Fig. 1), which shows that the simulation has been able to reproduce the main features. ${ }^{17)}$ Thus, the DEM simulations can be considered to be in general agreement with the experimental results. Thus, DEM modeling can be used to investigate the inter-particle percolation in burden descent, although the non-spherical coke particles are represented by spherical ones in the simulations. However, a number of parameters have to be set in the DEM simulations, and there are not always clear guidelines how to select the parameter values. Therefore, the following section presents a simulation study of the effect of particle properties on the extent of percolation, and provides, if possible, an explanation for why the effect arose.

\subsection{Effect of Parameters on the Simulation Results}

In order to express the extent of inter-particle percolation in the coke layer, the mass fraction of percolating particles (pellets) permeating into packing particles (coke) will be used as a variable. In the physical experiments, ${ }^{17)}$ the present authors applied a simplified procedure where first a single coke layer was studied at expansion of the device, noting its surface profile. Next, an expansion of the device with a pellet layer on top of a coke layer was undertaken, and the vertical difference between the pellet surface profile and the earlier determined coke profile was used to estimate the share of non-percolating pellets. In DEM simulations, such as those reported in the present study, it is possible to follow each and every particle, so the fraction was defined as

Table 2. Experimental and simulated total bed heights at four dimensional bed widths ( $c f$. Fig. 1) at different expansions of the device. ${ }^{17}$

\begin{tabular}{ccccccccc}
\hline & \multicolumn{7}{c}{ Dimensionless width } \\
\cline { 2 - 9 } \begin{tabular}{c} 
Bed expansion $(\mathrm{mm})$ \\
\cline { 2 - 9 }
\end{tabular} & \multicolumn{2}{c}{$1 / 8$} & \multicolumn{2}{c}{$3 / 8$} & \multicolumn{2}{c}{$5 / 8$} & \multicolumn{2}{c}{$7 / 8$} \\
\cline { 2 - 9 } & Exp. & DEM & Exp. & DEM & Exp. & DEM & Exp. & DEM \\
\hline 0 & 205 & 203 & 206 & 203 & 206 & 203 & 207 & 203 \\
50 & 199 & 198 & 195 & 196 & 187 & 185 & 166 & 160 \\
100 & 191 & 184 & 175 & 176 & 163 & 164 & 138 & 128 \\
150 & 179 & 180 & 152 & 155 & 145 & 133 & 128 & 116 \\
200 & 168 & 165 & 137 & 140 & 131 & 123 & 113 & 105 \\
\hline
\end{tabular}

$$
f=\frac{K_{\text {pe,perc }}}{K_{\text {pe,tot }}}
$$

i.e., as the ratio between the number of percolated pellets and the total number of pellets, since the pellets are of uniform size in the simulations. In Eq. (3), $K_{\text {pe,perc }}$ is obtained by noting the number of pellets that have entered below the upper surface of the coke-pellet interface. Thus, the larger the mass fraction, the more serious inter-particle percolation has occurred: A fraction of unity would mean that all pellets have permeated into the coke layer, while a value of zero that the two layers have stayed unmixed in the whole process. Figure 4 illustrates the evolution of the mass fraction with respect to the dimensionless bed expansion, $x=(l-$ $315 \mathrm{~mm}) / 200 \mathrm{~mm}$, where $l$ is the expansion of the bed, using the base setup of the present study. In the examples of the following subsections, the sensitivity of model parameters on the estimated pellet percolation is studied by changing one parameter at a time, keeping all other parameters at their base values reported in Table 1 .

\subsubsection{Coefficient of Restitution}

First, the effects of the coefficients of inter-particle restitution are studied. There are no evident effects of coefficient of inter-particle restitution of coke, except a minor influence at the highest expansions $x>0.7$ (which corresponds to a bed width $>450 \mathrm{~mm}$ ). The result is logical since the restitution of the packing (coke) particles should not influence the percolation of the pellets. By contrast to the above coefficient, the inter-particle restitution coefficient of pellet and coke (left panel of Fig. 5) has some effect on the percolation for $x>0.3$ : With an increased restitution coefficient, the mass fraction of percolated particles increases clearly, espe-

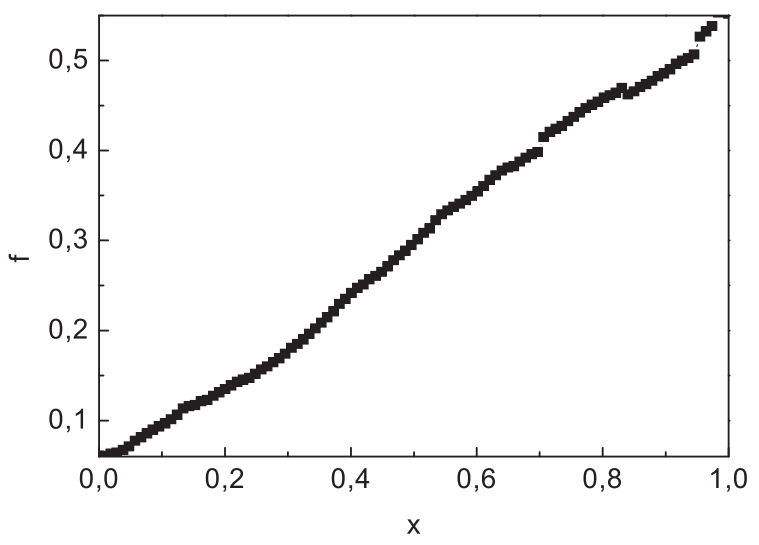

Fig. 4. Mass fraction of percolated pellets $(f)$ as a function of dimensionless expansion $(x)$ of the experimental device.
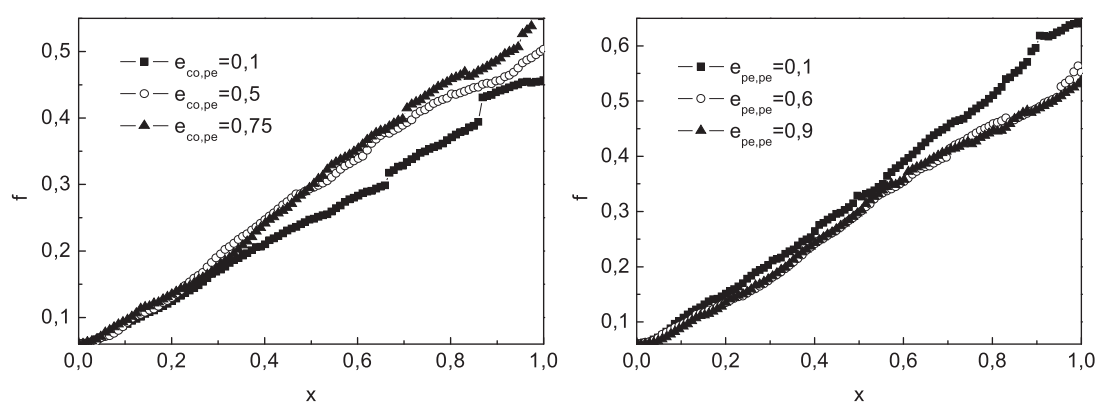

Fig. 5. Effect of inter-particle restitution coefficients $(e)$ on the percolation behavior. 
cially for larger expansions $(x>0.6)$. Thus, if the pellets bounce more they can more easily find their way into the space between coke particles, giving an increase in $f$. This observation is consistent with the results of the study of Lomine and Oger:"6) Pellets with higher restitution coefficients spend less time to percolate through coke layer, which means that the total number of pellet particles that percolate throughout the coke layer to the bottom plate, depicted in Fig. 6, is higher. As for the inter-particle restitution coefficient of pellets, the effect is opposite that of coefficient of coke and pellet: a low value of the coefficient makes the mass fraction percolated particles increase. A likely reason is that pellets with low restitution coefficient can easily percolate into the space between coke particles in linear form or block form. There is, however, a similarity between the effects of restitution coefficients of coke and pellet or pellet and pellet in that for high restitution coefficients the effect of an increase in the coefficients is little. It should, finally,

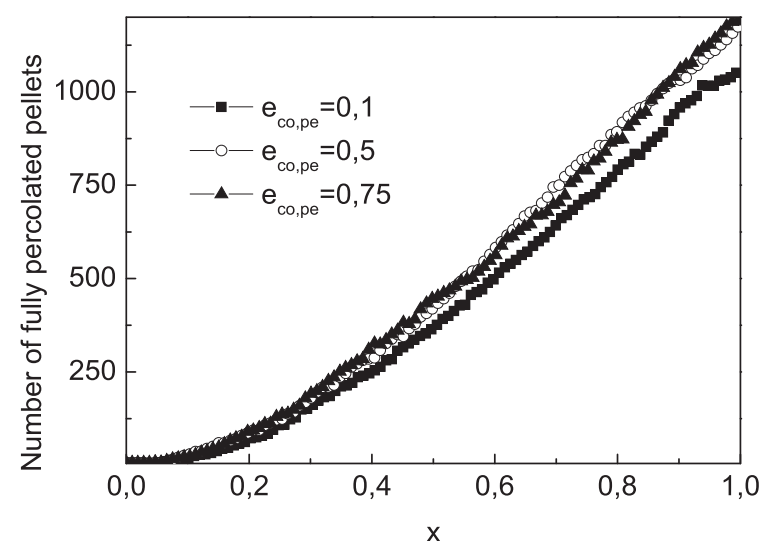

Fig. 6. Total number of pellet particles that have percolated to the bottom plate for different inter-particle restitution coefficients $(e)$ of pellet and coke. be noted that in experiments not reported in this paper the restitution coefficient for particle-wall had no clear effect on the percolation behavior.

In summary, a low inter-particle restitution coefficient of pellet and coke, and a high inter-particle restitution coefficient of pellets are helpful to decrease the inter-particles percolation, and the effects of restitution coefficients of coke and coke, coke and wall or pellet and wall are not notable.

\subsubsection{Particle Density}

Next, the role of the particle densities was studied: Figure 7 shows the mass fraction of percolated pellets for three particle densities of coke $\left(500 \mathrm{~kg} / \mathrm{m}^{3}, 1050 \mathrm{~kg} / \mathrm{m}^{3}\right.$, and $1500 \mathrm{~kg} / \mathrm{m}^{3}$, cf. left panel) and of pellet $\left(1000 \mathrm{~kg} / \mathrm{m}^{3}\right.$, $2284 \mathrm{~kg} / \mathrm{m}^{3}$, and $3000 \mathrm{~kg} / \mathrm{m}^{3}, c f$. right panel). It is seen that coke density of $1050 \mathrm{~kg} / \mathrm{m}^{3}$ results in strongest percolation, while the lightest coke gives a clearly lower percolation at full expansion of the device. A reason may be that the percolating pellets are able to shift the lightest coke, thus sealing the voids in the coke bed. As for the pellet density, this variable has no clear effect on the extent of percolation.

\subsubsection{Static Friction}

Figure 8 depicts the effects of inter-particle static friction for pellet-pellet and pellet-coke. Clearly, low values of the static friction coefficients $\left(e . g ., \mu_{\mathrm{s}}=0.1\right)$ promote percolation, but at large values $\left(\mu_{\mathrm{s}}=0.5\right)$ the value of the friction coefficient is of little importance for the percolating behavior. It is logical that low static friction means low resistance when pellet particles permeate through the coke layer, and that the resistance grows with the friction, but as a threshold value is exceeded other factors will become limiting for the percolation.

The effect of the static friction between the particles and the wall (left inclined moving and right perpendicular wall,
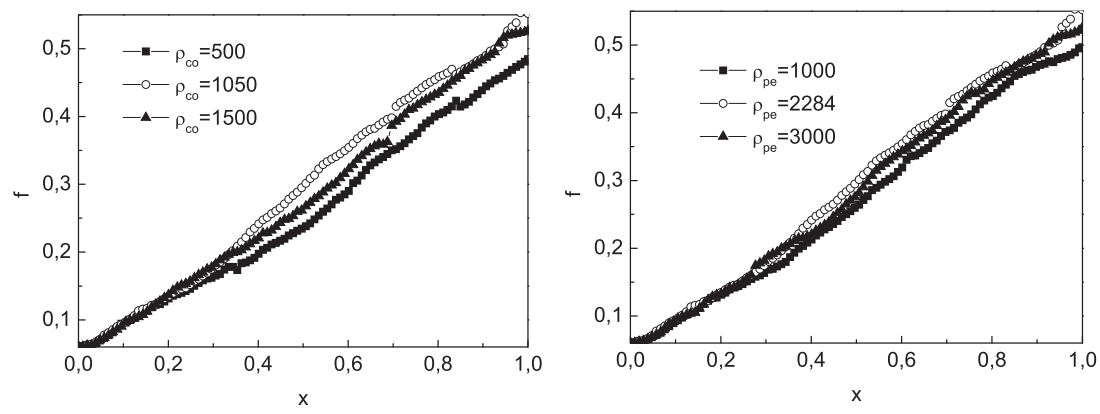

Fig. 7. Effect of particle density $\left(\rho\right.$ expressed in $\left.\mathrm{kg} / \mathrm{m}^{3}\right)$ on the percolation behavior.
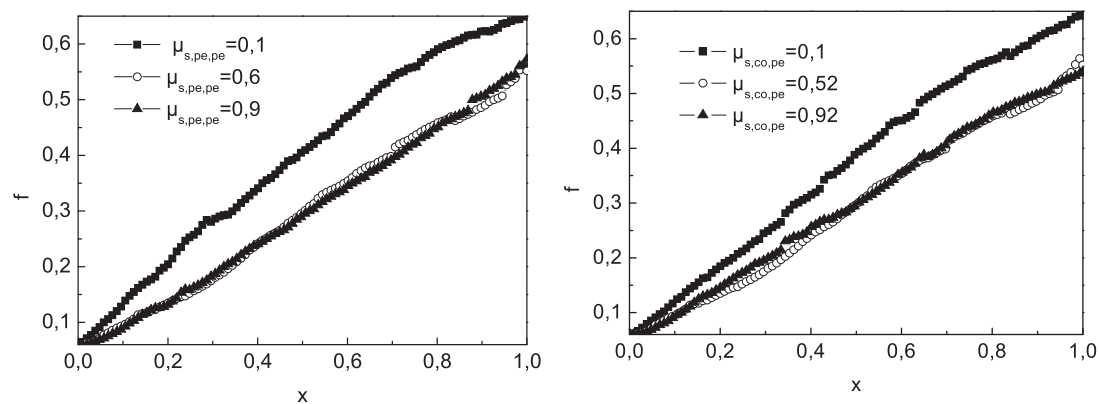

Fig. 8. Effect of inter-particle static friction $\left(\mu_{\mathrm{s}}\right)$ for pellet-pellet (left panel) and pellet-coke (right panel) on pellet percolation. 

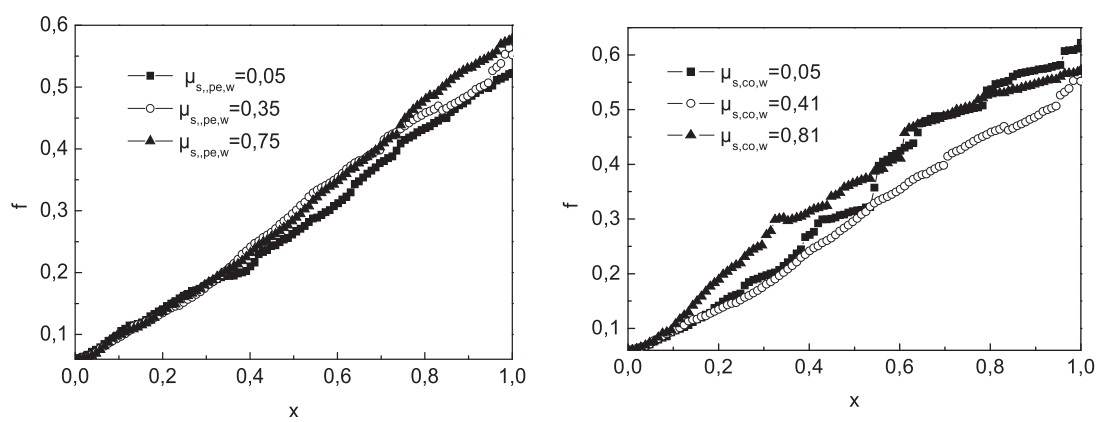

Fig. 9. Effect of static friction $\left(\mu_{\mathrm{s}}\right)$ between particles and wall for pellet-wall (left panel) and coke-wall (right panel) on the mass fraction of percolated pellets.
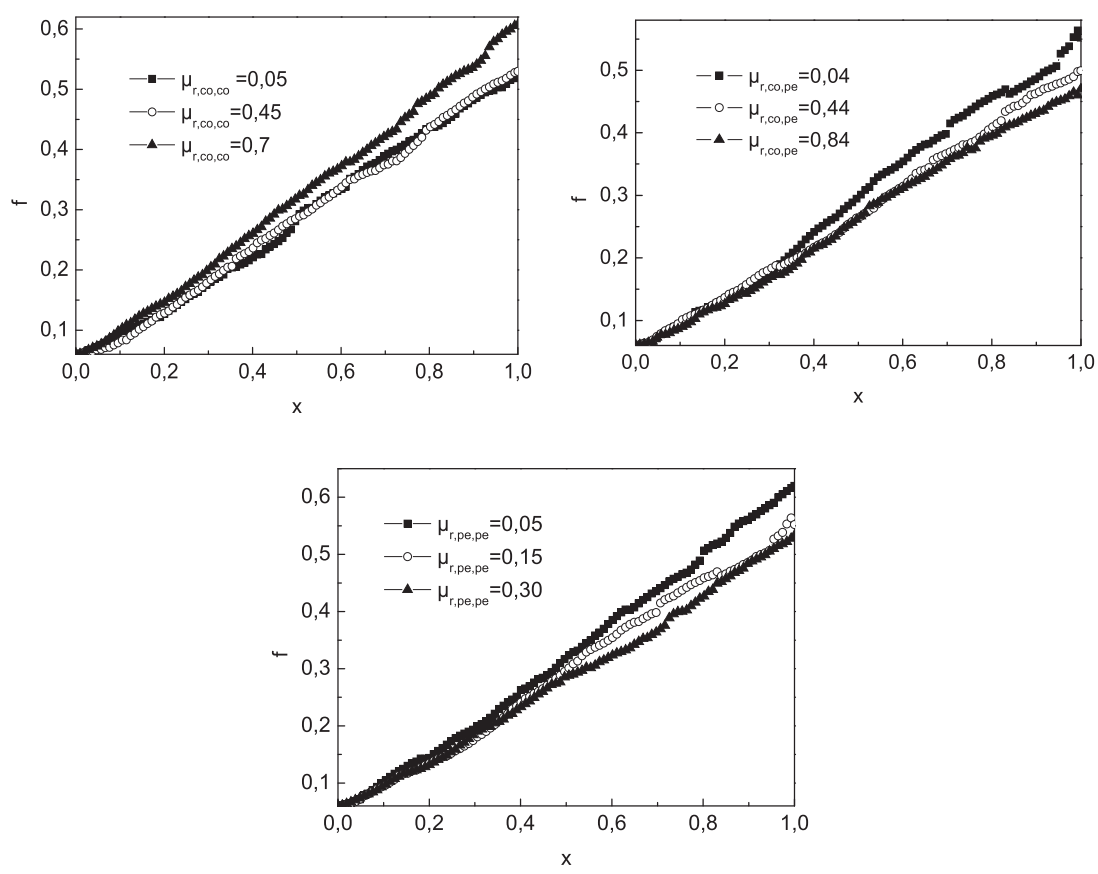

Fig. 11. Effect of inter-particle rolling friction $\left(\mu_{\mathrm{r}}\right)$ on the percolation behavior for pellet-wall (left panel) and coke-wall (right panel).

as well as wall behind the bed) is next illustrated (Fig. 9). With the increase of static friction between pellets and the wall, the mass fraction of percolated particles slightly increases for expansions $x>0.5$. For coke, in turn, the intermediate value of the friction coefficient gives the lowest percolation, while the behavior for a low friction coefficient is irregular. As for the static friction between coke and the rubber of the bottom plate, when the friction coefficient is low the whole coke layer moves as shown in the top panel of Fig. 10, and pellets at the fixed and moving walls percolate strongly into the coke layer. For high value of the static friction between coke and bottom plate (bottom panel), only the coke particles in the lowest part of the layer follow the moving wall, and the pellets roll in this direction and form a surface with a steep inclination. From Fig. 10 it is observed that the static friction coefficient mainly controls the layer distribution, but not the extent of percolation.

\subsubsection{Rolling Friction}

Figure 11 illustrates the effect of inter-particle rolling friction on the percolation. For small rolling friction between the coke particles, the variable does not affect the results significantly, but as high values are reached the per-

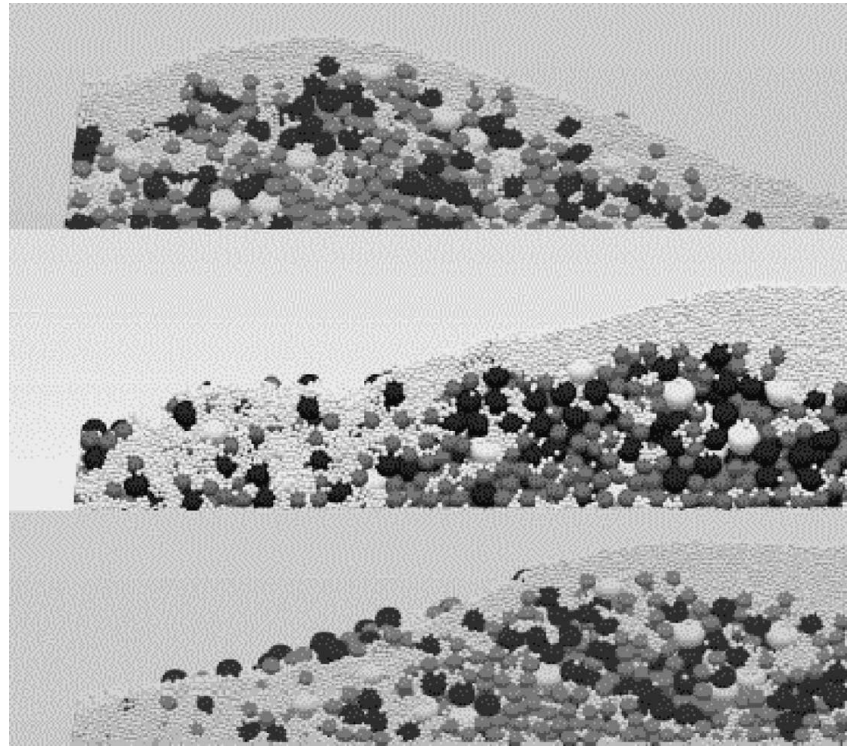

Fig. 10. Effect of static friction between coke and the moving bottom plate (top panel $\mu_{\mathrm{s}, \mathrm{co}, \mathrm{b}}=0.05$, middle panel $\mu_{\mathrm{s}, \mathrm{co}, \mathrm{b}}=$ 0.41 , and bottom panel $\mu_{\mathrm{s}, \mathrm{co}, \mathrm{b}}=0.81$ ) on the percolation behavior at maximum expansion of the device (Coke1: dark gray, Coke2: black, Coke3: white, Pellets: light gray.). 
colation gets stronger. This can be explained by the fact that a high rolling friction increases the angular velocity for coke particles ( $c f$. Fig. 12), and their rotation opens more void, which makes it easier for the pellets to permeate. The rolling friction between coke and pellets (Fig. 11) has little effect on the percolation, but a very low friction promotes percolation. A similar behavior is observed for the rolling friction between the pellets.

\subsubsection{Pellet Diameter}

The effect of pellet diameter on the percolation behavior is shown in Fig. 13 for three different cases: Table 1 reports the number of pellets in these cases. With an increase in diameter from $D_{\mathrm{pe}}=2 \mathrm{~mm}$, the mass fraction of percolated pellets decreases drastically, even though the pellet is clearly smaller than the coke. The difference in percolation between pellets of diameters $3 \mathrm{~mm}$ and $4 \mathrm{~mm}$ is, however, rather small. It should be noted that the condition for $D_{\mathrm{pe}}=4 \mathrm{~mm}$ still exceeds the critical ratio ${ }^{6,29)}$ of bed (coke) particle diameter to percolating (pellet) particle diameter, which expresses the lowest value of $D_{\mathrm{co}} / D_{\text {pe }}$ at which the smaller particle can drain through the bed. The results indicate that increasing the size of the percolating particles is a feasible method to reduce inter-particle percolation. Another observation is that with the increase in diameter, the relationship between

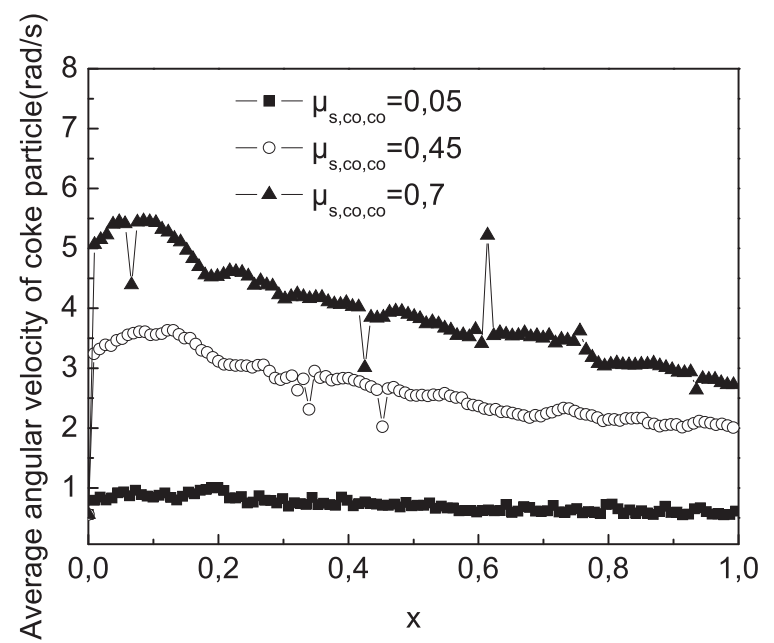

Fig. 12. Effect of inter-particle rolling friction of coke $\left(\mu_{\mathrm{r}, \mathrm{co}, \mathrm{co}}\right)$ on angular velocity of coke particles.

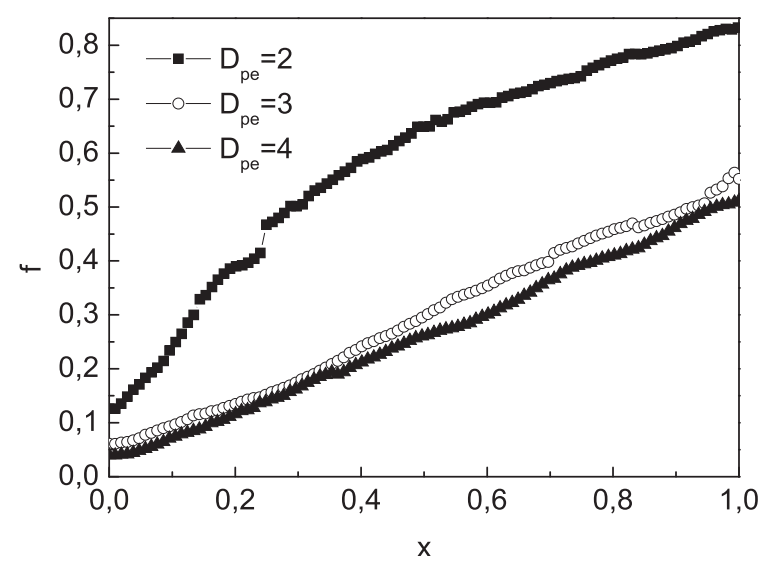

Fig. 13. Effect of pellet diameter $\left(D_{\mathrm{pe}}\right.$ in $\left.\mathrm{mm}\right)$ on the percolation behavior. the mass fraction of percolating particles and time (or bed expansion) becomes practically linear.

\subsubsection{Coke Shape}

The effect of the shape of the bed (coke) particles was also analyzed, using seven different shapes, and as the fourth case a combination of different shapes (with equal mass fraction), as indicated by the small inserts in Fig. 14. Somewhat surprisingly, the results show that the mass fraction of percolated pellets is lowest for cylindrical coke particles (squares), while it is highest for spherical coke particles (open stars). The hexahedron-shaped coke (circles) gives somewhat lower percolation than the spheres, while the irregular coke (normal triangles) initially gives almost identical pellet percolation as the cylindrical coke, but at higher expansion the percolation increases dramatically. As expected, the most regular shapes (spheres and cylinders)

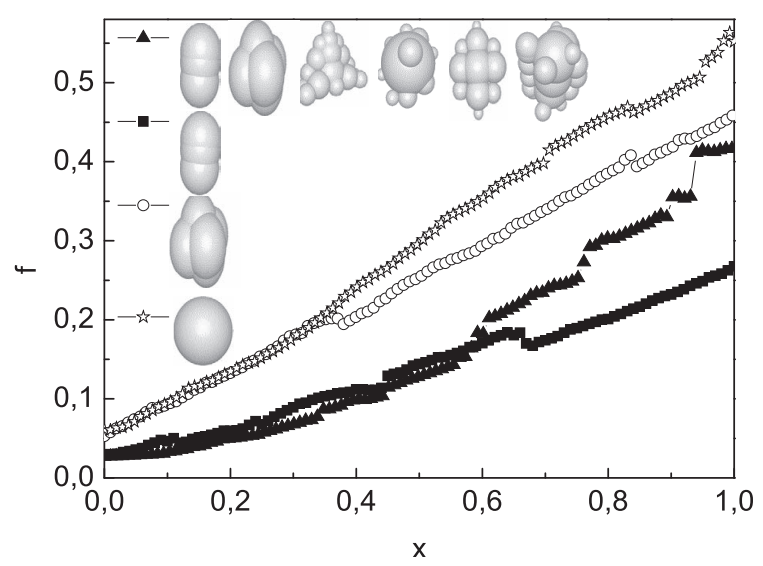

Fig. 14. Effect of coke shape on the mass fraction of percolating pellets, using the shapes depicted in the small inserts.

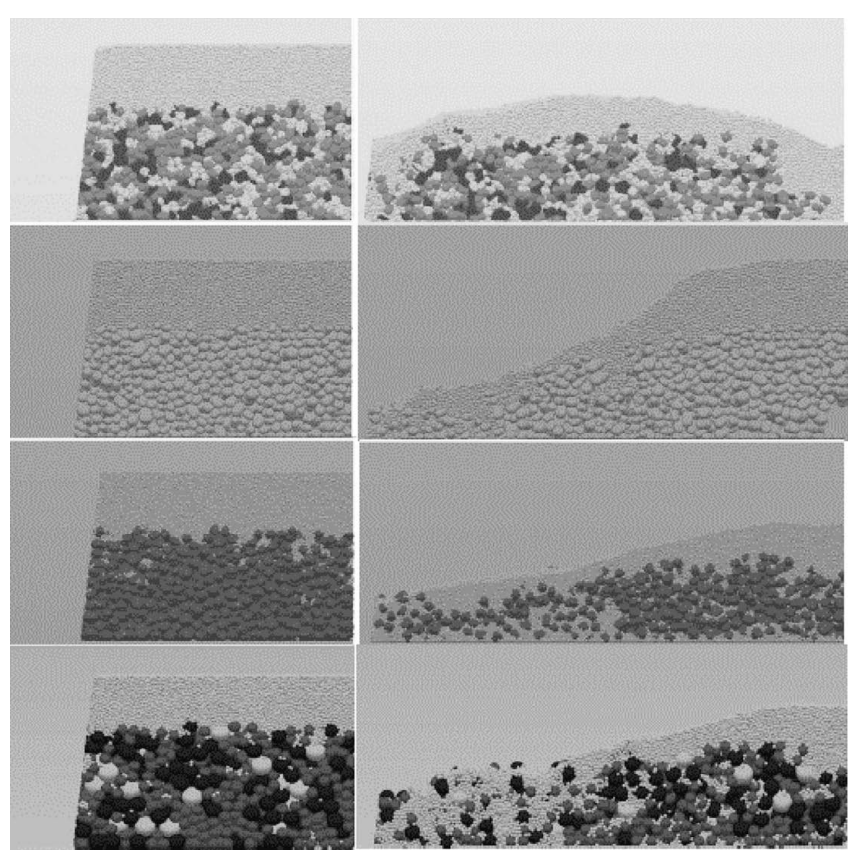

Fig. 15. Burden distribution at no (left panels) and full (right) expansion for coke of different shapes. The coke shapes appear in the same order as in the inserts in Fig. 14 (Coke1: dark gray, Coke2: black, Coke3: white, Pellets: light gray.). 
give rise to a rather smooth evolution of the percolation, while the more irregular shapes case abrupt changes in the percolation. Thus, the spherical bed particles do no lock the void in the bed void which allows for steady percolation.

The fundamentally different states that the differentshaped coke particles give rise to are illustrated in Fig. 15, where the initial states and the states at final expansion of the bed are illustrated. The panels denote the results for coke shapes in the same order as the inserts in Fig. 14 (i.e., distributed shapes at the top and spheres at the bottom). For coke of a complex shape (top panel in Fig. 15), the pellets will mainly percolate at the two edges (left and the right), which demonstrates that coke shape may influence the burden profile. The pellet percolation into a layer of cylindrical coke particles (second panels from the top) is constrained to the top part of the coke layer. This seems to indicate that the cylindrical coke particles more easily form impermeable densely packed thin layers.

Some tests were also undertaken to evaluate the effect of the expansion speed of the device (using bottom plate velocities of $w=\mathrm{d} l / \mathrm{d} t=1 \mathrm{~mm} / \mathrm{s}, 2 \mathrm{~mm} / \mathrm{s}$ and $4 \mathrm{~mm} / \mathrm{s}$ ), shear modulus of coke, pellet or wall (in the range $G=10^{6} \ldots 10^{9} \mathrm{~Pa}$ ) but these factors did only marginally affect the percolation behavior in the systems considered here.

\section{Conclusions}

This work has applied discrete element method (DEM) and small-scale experiments to study the pellet percolation behavior in a coke layer during burden descent in the blast furnace. The key objectives of the work were to validate the computational model with experimental data from a smallscale model, and to investigate the effect on percolation of particle properties, interaction coefficients and bed expansion.

Comparison of the simulation results with findings from the experiments showed that the computational model was able to reproduce the overall percolation behavior. However, the model slightly under-predicted the extent of percolation. This may be due to the differences of size distribution of coke and pellet in the simulations and in the experiments, the effect of the irregular coke shape, as well as differences in true and assumed friction coefficients between particles and wall. The study still shows that validation of the DEM models is feasible using small-scale experimental systems, and that DEM holds promise to accurately model complex flow mechanisms in particulate systems.

A number of particle properties were found to affect the percolation. The extent of percolation was influenced mainly by coke shape, pellet diameter (i.e., the ratio of percolating particles to bed particles), but also by inter-particle rolling friction, static friction and restitution. The rate of expansion of the device, the particle-wall rolling friction, the density of pellets and the shear moduli of coke, pellet and wall were found to have little effect. Some explanations for the underlying reasons for these observations were also provided. These findings may provide useful information in the design of charging program and new particulate materials for blast furnace burden in order to suppress or enhance the percolation of particles, e.g., in controlling the gas flow and bed permeability in the lumpy and cohesive zones of the fur- nace.

\section{Acknowledgements}

The work was undertaken within the ELEMET research program under FIMECC Ltd. We gratefully acknowledge financial support from Tekes and participating companies. The discrete element method (DEM) simulations and analysis were conducted using EDEM ${ }^{\circledR}$ particle simulation software provided by DEM Solutions Ltd., Edinburgh, Scotland, UK.

\section{Nomenclature}

$e$ coefficients of restitution (-)

$E$ Young modulus $(\mathrm{Pa})$

$f$ mass fraction of percolated pellets $(-)$

F force between particles $(\mathrm{N})$

g gravity acceleration $\left(\mathrm{m} / \mathrm{s}^{2}\right)$

$G$ shear modulus $(\mathrm{Pa})$

$I$ moment of inertia of particle $i\left(\mathrm{~kg} / \mathrm{m}^{2}\right)$

$k$ contact coefficient (-)

$K$ number of particles in contact with particle

$m$ mass of particle $(\mathrm{kg})$

n normal vector (from $i$ th to $j$ th particle)

$N$ number of particles

$R$ particle radius (m)

$t$ time (s)

$\mathbf{T}$ torque on particle $(\mathrm{Nm})$

u velocity of particle $(\mathrm{m} / \mathrm{s})$

$w$ expansion velocity of device $(\mathrm{mm} / \mathrm{s})$

$x$ dimensionless expansion of device (-)

\section{Greek letters}

$\delta$ deformation (m)

$\varepsilon$ restitution coefficient (-)

$\eta$ damping coefficient (-)

$\mu$ friction coefficient (-)

$v$ Poisson ratio $(-)$

$\rho$ density $\left(\mathrm{kg} / \mathrm{m}^{3}\right)$

$\omega$ angular velocity $(\mathrm{rad} / \mathrm{s})$

$\omega^{\prime}$ normalized angular velocity vector of the object at the contact point $(-)$

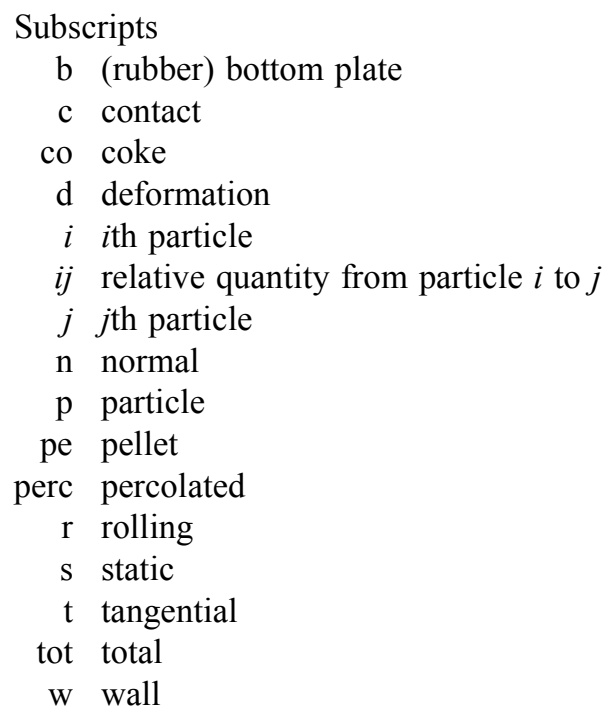




\section{REFERENCES}

1) J. Bridgwater, N. W. Sharpe and D. C. Stocker: Trans. Inst. Chem. Eng., 47 (1969), T114.

2) J. Bridgwater and N. D. Ingram: Trans. Inst. Chem. Eng., 49 (1971), 163.

3) A. M. Scott and J. Bridgwater: Ind. Eng. Chem. Fund., 14 (1975), 22

4) A. K. Jha and V. M. Puri: Powder Tech., 197 (2010), 274.

5) P. Richard, L. Oger, J. Lemaître, L. Samson and N. N. Medvedev: Granular Matter, 1 (1999), 203.

6) F. Lomine and L. Oger: J. Stat. Mech.: Theory Exp., July, (2006), P07019.

7) M. Rahman, H. P. Zhu, A.-B. Yu and J. Bridgwater: Particuology, 6 (2008), 475.

8) H. P. Zhu, M. Rahman, A.-B. Yu, J. Bridgwater and P. Zulli: Miner. Eng., 22 (2009), 961.

9) J. Li, A.-B. Yu, J. Bridgwater and S. L. Rough: Powder Tech., 203 (2010), 397.

10) M. Ichida, M. Takao, K. Kunitomo, S. Matsuzaki, T. Deno and K. Nishihara: ISIJ Int., 36 (1996), 493.

11) K. Narita, S. Inaba, M. Shimizu, A. Yamaguchi, I. Kobayashi and K. Okimoto: Trans. Iron Steel Inst. Jpn., 21 (1981), 405.

12) H. Takahashi, M. Tanno and J. Katayama: ISIJ Int., 36 (1996), 1354.

13) Y. Kajiwara, T. Jimbo and T. Sakai: Trans. Iron Steel Inst. Jpn., 23 (1983), 1045

14) M. Ichida, K. Nishihara, K. Tamura and M. Sugata: ISIJ Int., 31 (1991), 505.

15) H. Saxén and J. Hinnelä: Min. Proc. Extr. Met. Rev., 25 (2004), 1.

16) H. Mio, K. Yamamoto, A. Shimosaka, Y. Shirakawa and J. Hidaka: ISIJ Int., 47 (2007), 1745.

17) Y.-W. Yu and H. Saxén: ISIJ Int., 51 (2011), 1050.

18) P. A. Cundall and O. D. L. Strack: Geotech., 29 (1979), 47.

19) Z.-Y. Zhou, H.-P. Zhu, A.-B. Yu, B. Wright and P. Zulli: Comput. Chem. Eng., 32 (2008), 1760.

20) T. Faug, M. Naaim, D. Bertrand, P. Lachamp and F. Naaim-Bouvet: Surv. Geophys., 24 (2003), 555.

21) J. Zhang, Z. Hu, W. Ge, Y. Zhang, T. Li and J. Li: Ind. Eng. Chem. Res., 43 (2004), 5521.

22) Y. Tsuji, T. Tanaka and T. Ishida: Powder Tech., 71 (1992), 239.

23) A. D. Renzo and F. D. P. Maio: Chem. Eng. Sci., 59 (2004), 525.

24) C. Crowe, M. Sommerfeld and Y. Tsuji: Multiphase flows with droplets and particles. In: Multiphase flows with droplets and particles, CRC press, Boca Raton, Florida, (1998).

25) http://www.dem-solutions.com

26) Y.-W. Yu and H. Saxén: Chem. Eng. Sci., 65 (2010), 5237.

27) Y.-W. Yu and H. Saxén: Adv. Powder Tech., 22 (2011), 324.

28) H. Mio, S. Komatsuki, M. Akashi, A. Shimosaka, Y. Shirakawa, J. Hidaka, M. Kadowaki, S. Matsuzaki and K. Kunitomo: ISIJ Int., 49 (2009), 479.

29) J. A. Dodds: J. Coll. Int. Sci., 77 (1980), 317.

\section{Appendix}

The expressions for the forces and torques in Eqs. (1), (2) are as follows: $:^{20-27)}$

For the normal contact force we have

$$
\mathbf{F}_{\mathrm{cn}, i j}=-k_{\mathrm{n}} \delta_{\mathrm{n}}^{3 / 2} \mathbf{n} ; \quad k_{\mathrm{n}}=\frac{4}{3} \sqrt{R_{i j}} E^{*}
$$

where $\delta_{\mathrm{n}}=\left|\delta_{\mathrm{n}, i j}\right|$ and the normal vector is calculated from the normal deformation, $\delta_{\mathrm{n}, i j}$, as

$$
\mathbf{n}=\delta_{\mathrm{n}, i j} /\left|\delta_{\mathrm{n}, i j}\right|
$$

and the effective radius, $R_{i j}$, and the equivalent elastic modulus, $E^{*}$, are given by

$$
R_{i j}=\frac{R_{i} R_{j}}{R_{i}+R_{j}} ; \quad E^{*}=\left[\frac{1-v_{i}^{2}}{E_{i}}+\frac{1-v_{j}^{2}}{E_{j}}\right]^{-1}
$$

where $E$ and $v$ are the Young modulus and the Poisson ratio of the particles, respectively.

The normal damping force is written as

$$
\begin{aligned}
& \mathbf{F}_{\mathrm{dn}, i j}=-\eta_{\mathrm{n}} \mathbf{u}_{\mathrm{n}, i j} ; \quad \eta_{\mathrm{n}}=2 \sqrt{\frac{5}{6}} \frac{\ln e}{\sqrt{\ln ^{2} e+\pi^{2}}} \sqrt{2 E^{*} m_{i j} \sqrt{R_{i j} \delta_{n}}} ; \\
& m_{i j}=\frac{m_{i} m_{j}}{m_{i}+m_{j}} \ldots \ldots \ldots \ldots \ldots \ldots \ldots \ldots \ldots \ldots \ldots \ldots \ldots \ldots \ldots \ldots \ldots \ldots \ldots \ldots \ldots \ldots \ldots \ldots \ldots \ldots \ldots \ldots \ldots \ldots \\
&\text { (A } 4 \mathrm{a}, \mathrm{b}, \mathrm{c})
\end{aligned}
$$

where $e$ expresses the coefficient of restitution. The velocity in the normal and tangential directions are

$$
\mathbf{u}_{\mathrm{n}, i j}=\left(\mathbf{u}_{i j} \cdot \mathbf{n}\right) \cdot \mathbf{n} ; \mathbf{u}_{\mathrm{t}, i j}=\mathbf{u}_{i j}-\mathbf{u}_{\mathrm{n}, i j}
$$

with

$$
\mathbf{u}_{i j}=\mathbf{u}_{j}-\mathbf{u}_{i}+\omega_{j} \times R_{j} \mathbf{n}-\omega_{i} \times R_{i} \mathbf{n}
$$

In the tangential direction, the contact force is

$$
\mathbf{F}_{\mathrm{ct}, i j}=-k_{\mathrm{t}} \delta_{\mathrm{t}} ; \quad k_{\mathrm{t}}=8 G_{i j} \sqrt{R_{i j} \delta_{\mathrm{n}}} ; \quad G_{i j}=\frac{G_{i} G_{j}}{G_{i}+G_{j}} \ldots .(\mathrm{A} 7 \mathrm{a}, \mathrm{b}, \mathrm{c})
$$

where $\delta_{\mathrm{t}}$ is the tangential overlap and $G$ denotes the shear modulus of the particle, while the damping force is given by

$$
\mathbf{F}_{\mathrm{dt}, i j}=-\eta_{\mathrm{t}} \mathbf{u}_{\mathrm{t}, i j} ; \quad \eta_{\mathrm{t}}=2 \sqrt{\frac{5}{6}} \frac{\ln e}{\sqrt{\ln ^{2} e+\pi^{2}}} \sqrt{8 G_{i j} \sqrt{R_{i j} \delta_{n}} m_{i j}}
$$

under the condition

$$
\mathbf{F}_{\mathrm{ct}, i j}+\mathbf{F}_{\mathrm{dt}, i j} \leq \mu_{\mathrm{s}} \mathbf{F}_{\mathrm{cn}, i j}
$$

where $\mu_{\mathrm{s}}$ is the static friction. Thus, in Eq. (1) the sum of the two terms in the left-hand side of Eq. (A9) cannot exceed the static contact friction force, $\mu_{\mathrm{s}} \mathbf{F}_{\mathrm{cn}, i j}$.

As for the rolling forces, the torque is given as the vector product

$$
\mathbf{T}_{\mathrm{t}, i j}=R_{i} \mathbf{n} \times\left(\mathbf{F}_{\mathrm{ct}, i j}+\mathbf{F}_{\mathrm{dt}, i j}\right)
$$

while the friction torque is given by

$$
\mathbf{T}_{\mathrm{r}, i j}=-\mu_{\mathrm{r}}\left|\mathbf{F}_{\mathrm{cn}, i j}\right| \omega^{\prime} ; \quad \omega^{\prime}=\omega_{i} /\left|\omega_{i}\right| \ldots . .(\mathrm{A} 11 \mathrm{a}, \mathrm{b})
$$

where $\mu_{\mathrm{r}}$ is the rolling friction. 